\title{
Comparative study of three-nucleon force models in nuclear matter
}

\author{
Domenico Logoteta, ${ }^{1}$ Isaac Vidaña, ${ }^{2}$ Ignazio Bombaci, ${ }^{1,3}$ and Alejandro Kievsky ${ }^{1}$ \\ ${ }^{1}$ INFN, Sezione di Pisa, Largo Bruno Pontecorvo 3, I-56127 Pisa, Italy \\ ${ }^{2}$ CFisUC, Department of Physics, University of Coimbra, PT-3004-516 Coimbra, Portugal \\ ${ }^{3}$ Dipartimento di Fisica, Universitá di Pisa, Largo Bruno Pontecorvo 3, I-56127 Pisa, Italy \\ (Received 4 February 2015; revised manuscript received 25 May 2015; published 15 June 2015)
}

\begin{abstract}
We calculate the energy per particle of symmetric nuclear matter and pure neutron matter using the microscopic many-body Brueckner-Hartree-Fock (BHF) approach and employing the Argonne V18 (AV18) nucleon-nucleon (NN) potential supplemented with two different three-nucleon force models recently constructed to reproduce the binding energy of ${ }^{3} \mathrm{H},{ }^{3} \mathrm{He}$, and ${ }^{4} \mathrm{He}$ nuclei as well as the neutron-deuteron doublet scattering length. We find that none of these new three-nucleon force models is able to reproduce simultaneously the empirical saturation point of symmetric nuclear matter and the properties of three- and four-nucleon systems.
\end{abstract}

DOI: 10.1103/PhysRevC.91.064001

PACS number(s): 26.60.Dd, 26.60.Kp, 97.60.Jd

\section{INTRODUCTION}

The important role played by the three-nucleon forces (TNFs) has been widely pointed out both in finite nuclei and nuclear matter calculations (see, e.g., [1-20] and references therein). First indications for the inclusion of a TNF in the nuclear Hamiltonian arose from the discrepancy between the results of the ${ }^{3} \mathrm{H}$ binding energy using different nucleonnucleon $(\mathrm{NN})$ potentials and its experimental value. For example using high precision NN potentials, able to fit $\mathrm{NN}$ scattering data up to an energy of $350 \mathrm{MeV}$ with a $\chi^{2}$ per datum close to 1 , the ${ }^{3} \mathrm{H},{ }^{3} \mathrm{He}$, and ${ }^{4} \mathrm{He}$ binding energies are underpredicted by about 1 and $4 \mathrm{MeV}$ in the case of the threeand four-nucleon systems respectively [21]. A commonly accepted solution to this problem has been the introduction of TNF that could bridge the gap between the calculated binding energy $[22,23]$ based on two-body interactions and the experimental binding energies. The origin of such a TNF lies in the fact that nucleons are treated as pointlike particles, disregarding therefore their internal quark structure.

In nuclear matter calculations based on nonrelativistic many-body approaches similar problems arise. In such calculations, when only a two-body NN potential is used, symmetric nuclear matter (SNM) results over-bound and its empirical saturation point $\rho_{0}=0.16 \mathrm{fm}^{-3}, E /\left.A\right|_{\rho_{0}}=-16 \mathrm{MeV}$ cannot be reproduced. As in the case of few-nucleon systems, also for the nuclear matter case TNFs are considered as the missing physical effect of the whole picture. In addition, TNFs are likely crucial in the case of dense $\beta$-stable nuclear matter to obtain a stiff equation of state (EOS) [24-27] compatible with the measured masses $M=1.97 \pm 0.04 M_{\odot}$ [28] and $M=$ $2.01 \pm 0.04 M_{\odot}[29]$ of the neutron stars in PSR J1614-2230 and PSR J0348+0432 respectively.

In relativistic microscopic approaches, such as the DiracBrueckner-Hartree-Fock one, the importance of three-nucleon interaction is diminished [30]. In this approach TNFs are partially included by means of nucleon-antinucleon virtual excitations in the scalar $\sigma$-meson exchange process due to the dressed Dirac spinor in the nuclear medium.

Although systems as nuclear matter and finite nuclei deal with the same interactions, the numerical calculations of the properties of these systems are commonly performed using different approaches and numerical techniques. Moreover, different parametrizations of the same TNF are most of the time present in literature according to the system treated: finite nuclei or nuclear matter. In fact, the TNF parameters can be fixed to reproduce the properties of few-nucleon $(A=3,4)$ systems, or the empirical saturation point of nuclear matter. In this paper we analyze the differences existing in the sector of three-nucleon interaction between these two areas of nuclear physics. We would like to see if the need to use different TNF parametrization is the consequence of a restricted search in the relative strength of some TNF terms or if it is a more fundamental problem. For example in Ref. [31] it has been shown that in order to simultaneously describe the ${ }^{3} \mathrm{H},{ }^{3} \mathrm{He}$, and ${ }^{4} \mathrm{He}$ binding energies and the neutron-deuteron $(n-d)$ doublet scattering length it is necessary to modify some of the strengths present in the TNF. Along this line we investigate the possibility to find a paramatrization of the TNF suitable both for finite nuclei and many-body calculations.

The paper is organized as follows: in the second section we present the three-nucleon force models used in this work; in the third section we briefly review the many-body BruecknerHartree-Fock (BHF) approach and we discuss how to include a TNF in this formalism; finally, the fourth section is devoted to show the results of our calculations and to outline the main conclusions of this study.

\section{THREE-NUCLEON FORCES}

The TNFs that we considered in this work are the new Tucson-Melbourne potential [32] (hereafter $\mathrm{TM}^{\prime}$ ) and the three-nucleon potential based on chiral perturbation theory calculated at next-to-next-to-leading order [33] in its local form [34] (hereafter N2LOL). The $\mathrm{TM}^{\prime}$ potential is a revisited version of the older Tucson-Melbourne potential [35] readjusted in order to satisfy the chiral symmetry. The final operatorial structure coincides with the one obtained in the old Brasilian three-nucleon model [36]. These potentials, in conjunction with the Argonne V18 NN potential [37], have been recently used by the Pisa group [31] to find a new parametrization able to reproduce simultaneously the binding energies of the ${ }^{4} \mathrm{He}$ and ${ }^{3} \mathrm{He}$ nuclei and the neutron-deuteron 


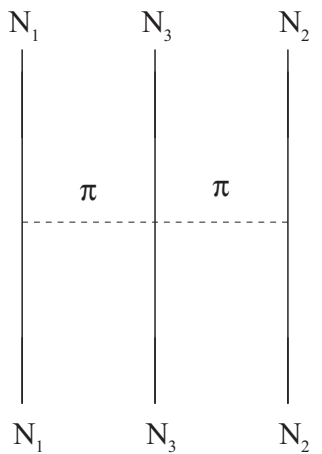

(a)

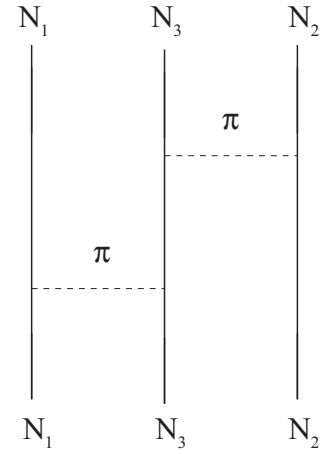

(b)

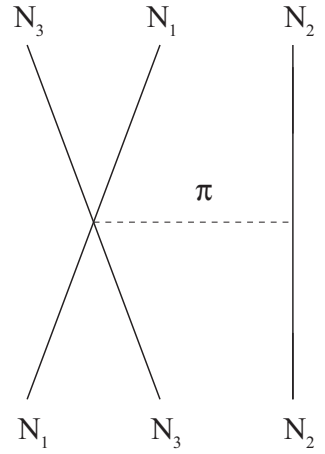

(c)

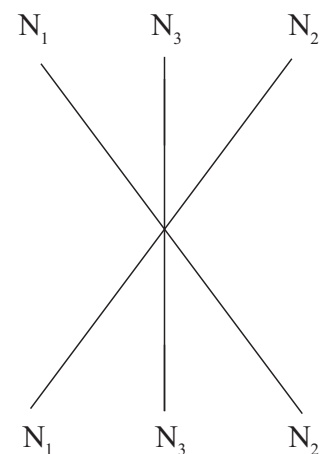

(d)

FIG. 1. Diagrams representing the contribution of terms $a$ [diagram (a)], $b, d$ [diagram (b)], $D$ [diagram (c)], and $E$ [diagram (d)] to the generic three-nucleon force $W(1,2,3)$ of Eq. (2).

scattering length ${ }^{2} a_{n d}$. In the following, we report in details the expressions for the $\mathrm{TM}^{\prime}$ and the N2LOL potentials [31]. Notice that we corrected a few misprints ${ }^{1}$ appearing in some of the equations for the TNF reported in [31]:

$$
W=\sum_{\text {cyc }} W(1,2,3) \text {, }
$$

where $W(1,2,3)$ is a generic term that can be put in the following form:

$$
\begin{aligned}
W(1,2,3)= & a W_{a}(1,2,3)+b W_{b}(1,2,3)+d W_{d}(1,2,3) \\
& +c_{D} W_{D}(1,2,3)+c_{E} W_{E}(1,2,3) .
\end{aligned}
$$

In Eq. (2) each term corresponds to one of the different mechanism shown in Fig. 1 and has a different operatorial structure. The numerical values of the constants $a, b, d, c_{D}$, and $c_{E}$ appearing in front of each term of Eq. (2) are given in Table I for each model. The first three terms arise from the exchange of two pions between the three nucleons [Fig. 1,

\footnotetext{
${ }^{1}$ In Eq. (12) of Ref. [31] the factor $\frac{1}{12 \pi^{2}}$ should be replaced by $\left(\frac{1}{12 \pi}\right)^{2}$; in Eq. (5) of Ref. [31] a factor $1 / 3$ should be added in the function $y(r)$ in the case of the $\mathrm{TM}^{\prime}$ potential [see the definition of the factor $\eta_{0}$ in Eq. (12) of our work]; in the expression for the strength $W_{0}$ for the $\mathrm{TM}^{\prime}$ model [see our Eq. (4)] the value $g^{2}=197.7$ reported in Ref. [31] should be replaced by $g^{2}=179.7(g=13.405)$. In Eq. (14) of Ref. [31], the strength $W_{0}^{E}$ in the case of the TM' model is not given by Eq. (12) of Ref. [31], but by Eq. (11) of the present work. We checked that when deriving the TNF constants for the TM' model (Table II of [31]), the authors of Ref. [31] used the correct expressions for $W_{0}$ and $W_{0}^{E}$.
}

TABLE I. Numerical values of the constants $a, b, d, c_{D}$, and $c_{E}$ appearing in front of each term of Eq. (2) for each model. Constants $c_{1}=-0.00081 \mathrm{MeV}^{-1}, c_{3}=-0.0032 \mathrm{Mev}^{-1}$, and $c_{4}=$ $-0.0054 \mathrm{MeV}^{-1}$ are taken from Ref. [38].

\begin{tabular}{lccccc}
\hline \hline & $a$ & $b$ & $d$ & $c_{D}$ & $c_{E}$ \\
\hline $\mathrm{TM}^{\prime}$ & $-0.87 / m_{\pi}$ & $-2.58 / m_{\pi}^{3}$ & $-0.753 / m_{\pi}^{3}$ & 0 & 0 \\
$\mathrm{~N} 2 \mathrm{LOL}$ & $c_{1} m_{\pi}^{2}$ & $c_{3} / 2$ & $c_{4} / 4$ & 1 & -0.029 \\
\hline
\end{tabular}

diagrams (a) and (b)]. The term $a$ comes from $\pi N S$-wave scattering [diagram (a)] whereas the terms $b$ and $d$, which are the most important, come from $\pi N P$-wave scattering [diagram (b)]. The specific form of these three terms in configuration space is the following:

$$
\begin{aligned}
W_{a}(1,2,3)= & -W_{0}\left(\boldsymbol{\tau}_{1} \cdot \boldsymbol{\tau}_{2}\right)\left(\boldsymbol{\sigma}_{1} \cdot \boldsymbol{r}_{31}\right)\left(\boldsymbol{\sigma}_{2} \cdot \boldsymbol{r}_{23}\right) y\left(r_{31}\right) y\left(r_{23}\right), \\
W_{b}(1,2,3)= & W_{0}\left(\boldsymbol{\tau}_{1} \cdot \boldsymbol{\tau}_{2}\right)\left[\left(\boldsymbol{\sigma}_{1} \cdot \boldsymbol{\sigma}_{2}\right) y\left(r_{31}\right) y\left(r_{23}\right)\right. \\
& +\left(\boldsymbol{\sigma}_{1} \cdot \boldsymbol{r}_{31}\right)\left(\boldsymbol{\sigma}_{2} \cdot \boldsymbol{r}_{23}\right)\left(\boldsymbol{r}_{31} \cdot \boldsymbol{r}_{23}\right) t\left(r_{31}\right) t\left(r_{23}\right) \\
& +\left(\boldsymbol{\sigma}_{1} \cdot \boldsymbol{r}_{31}\right)\left(\boldsymbol{\sigma}_{2} \cdot \boldsymbol{r}_{31}\right) t\left(r_{31}\right) y\left(r_{23}\right) \\
& \left.+\left(\boldsymbol{\sigma}_{1} \cdot \boldsymbol{r}_{23}\right)\left(\boldsymbol{\sigma}_{2} \cdot \boldsymbol{r}_{23}\right) y\left(r_{31}\right) t\left(r_{23}\right)\right], \\
W_{d}(1,2,3)= & W_{0}\left(\boldsymbol{\tau}_{3} \cdot \boldsymbol{\tau}_{1} \times \boldsymbol{\tau}_{2}\right)\left[\left(\boldsymbol{\sigma}_{3} \cdot \boldsymbol{\sigma}_{2} \times \boldsymbol{\sigma}_{1}\right) y\left(r_{31}\right) y\left(r_{23}\right)\right. \\
& +\left(\boldsymbol{\sigma}_{1} \cdot \boldsymbol{r}_{31}\right)\left(\boldsymbol{\sigma}_{2} \cdot \boldsymbol{r}_{23}\right)\left(\boldsymbol{\sigma}_{3} \cdot \boldsymbol{r}_{31} \times \boldsymbol{r}_{23}\right) t\left(r_{31}\right) t\left(r_{23}\right) \\
& +\left(\boldsymbol{\sigma}_{1} \cdot \boldsymbol{r}_{31}\right)\left(\boldsymbol{\sigma}_{2} \cdot \boldsymbol{r}_{31} \times \boldsymbol{\sigma}_{3}\right) t\left(r_{31}\right) y\left(r_{23}\right) \\
& \left.+\left(\boldsymbol{\sigma}_{2} \cdot \boldsymbol{r}_{23}\right)\left(\boldsymbol{\sigma}_{3} \cdot \boldsymbol{r}_{23} \times \boldsymbol{\sigma}_{1}\right) y\left(r_{31}\right) t\left(r_{23}\right)\right] .
\end{aligned}
$$

Terms $a, b$, and $d$ are present in both the $\mathrm{TM}^{\prime}$ and N2LOL models. The functions $y(r)$ and $t(r)$ are defined below. $W_{0}$ denotes the overall strength of these three terms and is defined in a different way in the two models. In the $\mathrm{TM}^{\prime}$ case $W_{0}$ reads

$$
W_{0}=\left(\frac{g m_{\pi}}{8 \pi m_{N}}\right)^{2} m_{\pi}^{4},
$$

where $g=13.405$, and $m_{\pi}$ and $m_{N}$ are the pion and nucleon masses, respectively. In the N2LOL model $W_{0}$ is given by

$$
W_{0}=\left(\frac{1}{12 \pi}\right)^{2}\left(\frac{m_{\pi}}{F_{\pi}}\right)^{4} g_{A}^{2} m_{\pi}^{2},
$$

with $F_{\pi}=92.4 \mathrm{MeV}$ and $g_{A}=1.29$.

Term $D$ is present only in the N2LOL model, and it provides the contribution of a two-nucleon contact term with the emission and absorption of a pion [Fig. 1 diagram (c)]. Its local form in configuration space, derived in Ref. [34] reads

$$
\begin{aligned}
W_{D}(1,2,3)= & W_{0}^{D}\left(\boldsymbol{\tau}_{1} \cdot \boldsymbol{\tau}_{2}\right)\left[( \sigma _ { 1 } \cdot \boldsymbol { \sigma } _ { 2 } ) \left[y\left(r_{31}\right) Z_{0}\left(r_{23}\right)\right.\right. \\
& \left.+Z_{0}\left(r_{31}\right) y\left(r_{23}\right)\right]+\left(\sigma_{1} \cdot \boldsymbol{r}_{31}\right)\left(\boldsymbol{\sigma}_{2} \cdot \boldsymbol{r}_{31}\right) t\left(r_{31}\right) \\
& \left.\times Z_{0}\left(r_{23}\right)+\left(\boldsymbol{\sigma}_{1} \cdot \boldsymbol{r}_{23}\right)\left(\boldsymbol{\sigma}_{2} \cdot \boldsymbol{r}_{23}\right) Z_{0}\left(r_{31}\right) t\left(r_{23}\right)\right],
\end{aligned}
$$


TABLE II. Three different parametrizations of the $\mathrm{TM}^{\prime}$ threebody force. The value $a=-0.87 m_{\pi}^{-1}$ has been kept fix in all the three cases. See text and Ref. [31] for details.

\begin{tabular}{lcccc}
\hline \hline & $b\left(m_{\pi}^{-3}\right)$ & $d\left(m_{\pi}^{-3}\right)$ & $c_{E}$ & $\Lambda\left(m_{\pi}\right)$ \\
\hline TM1 $^{\prime}$ & -8.256 & -4.690 & 1.0 & 4.0 \\
TM2 $^{\prime}$ & -3.870 & -3.375 & 1.6 & 4.8 \\
TM3 $^{\prime}$ & -2.064 & -2.279 & 2.0 & 5.6 \\
\hline \hline
\end{tabular}

where the constant $W_{0}^{D}$ is defined as

$$
W_{0}^{D}=\left(\frac{1}{12 \pi}\right)^{2}\left(\frac{m_{\pi}}{F_{\pi}}\right)^{4}\left(\frac{m_{\pi}}{\Lambda_{x}}\right) \frac{g_{A} m_{\pi}}{8},
$$

$\Lambda_{x}$ being the chiral symmetry breaking scale with a value of $700 \mathrm{MeV}$.

Term $E$ is also present only in the N2LOL model but not in the $\mathrm{TM}^{\prime}$ one. For the N2LOL model, term $E$ gives the contribution of a three-nucleon contact term [Fig. 1 diagram (d)]. It reads

$$
W_{E}^{\mathrm{N} 2 \mathrm{LOL}}(1,2,3)=W_{0}^{E}\left(\boldsymbol{\tau}_{1} \cdot \boldsymbol{\tau}_{2}\right) Z_{0}\left(r_{31}\right) Z_{0}\left(r_{23}\right),
$$

where $W_{0}^{E}$ is

$$
W_{0}^{E}=\left(\frac{1}{12 \pi}\right)^{2}\left(\frac{m_{\pi}}{F_{\pi}}\right)^{4}\left(\frac{m_{\pi}}{\Lambda_{x}}\right) m_{\pi} .
$$

Although, as said before, the term $E$ is not present in the original $\mathrm{TM}^{\prime}$ model, recently in Ref. [31] this model has been extended by introducing a term similar to that of Eq. (8):

$$
W_{E}^{\mathrm{TM}^{\prime}}(1,2,3)=W_{0}^{E} Z_{0}\left(r_{31}\right) Z_{0}\left(r_{23}\right),
$$

where, for simplicity, the isospin dependence has been omitted and the strength $W_{0}^{E}$ is in this case given by

$$
W_{0}^{E}=\left(\frac{g m_{\pi}}{8 \pi m_{N}}\right)^{2} \frac{m_{\pi}^{2}}{\Lambda_{x}} .
$$

We will refer to this modification of the original $\mathrm{TM}^{\prime}$ potential also as $\mathrm{TM}^{\prime}$ and it is the one that we will use in all the calculations presented in this work. Note that for this extended $\mathrm{TM}^{\prime}$ force [31] the constant $c_{E}$ is different from zero (see Table II) and not equal to zero (see Table I).

The radial dependence of the five terms is encoded in the functions $y(r), t(r)$, and $Z_{0}(r)$. For the $\mathrm{TM}^{\prime}$ and $\mathrm{N} 2 \mathrm{LOL}$ models, the functions $y(r)$ and $t(r)$ are

$$
y(r)=\eta_{0} \frac{f_{0}^{\prime}(r)}{r}, \quad t(r)=\frac{y^{\prime}(r)}{r},
$$

where the prime symbol in $f_{0}^{\prime}(r)$ and $y^{\prime}(r)$ denotes the derivative with respect to $r$, the factor $\eta_{0}$ is equal to $1 / 3$ for the $\mathrm{TM}^{\prime}$ model and to 1 for the N2LOL model. The function $f_{0}(r)$ is given by

$$
f_{0}(r)=\frac{12 \pi}{m_{\pi}^{3}} \frac{1}{2 \pi^{2}} \int_{0}^{\infty} d q q^{2} \frac{j_{0}(q r)}{q^{2}+m_{\pi}^{2}} F_{\Lambda}(q),
$$

with $j_{0}(q r)=\sin (q r) /(q r)$. The cutoff function $F_{\Lambda}(q)$ in the $\mathrm{TM}^{\prime}$ model is taken as

$$
F_{\Lambda}(q)=\left[\frac{\Lambda^{2}-m_{\pi}^{2}}{\Lambda^{2}+q^{2}}\right]^{2},
$$

while in the N2LOL model it is given by

$$
F_{\Lambda}(q)=\exp \left(-q^{4} / \Lambda^{4}\right) .
$$

$\Lambda$ is a momentum cutoff parameter that fixes the scale of the system in momentum space. In the N2LOL, it has been set to $\Lambda=500 \mathrm{MeV}$, whereas in the $\mathrm{TM}^{\prime}$ model the ratio $\Lambda / m_{\pi}$ has been varied in order to describe the ${ }^{3} \mathrm{H}$ and ${ }^{4} \mathrm{He}$ binding energies at fixed values of the constants $a, b$, and $d$. In literature the $\mathrm{TM}^{\prime}$ potential has been used in several works (see e.g., Ref. [39]) with typical values around $\Lambda=5 m_{\pi}$.

The function $Z_{0}(r)$ appearing in Eqs. (6), (8), and (10) is defined as

$$
Z_{0}(r)=\frac{12 \pi}{m_{\pi}^{3}} \frac{1}{2 \pi^{2}} \int_{0}^{\infty} d q q^{2} j_{0}(q r) F_{\Lambda}(q),
$$

with $F_{\Lambda}(q)$ defined in Eq. (14) for the $\mathrm{TM}^{\prime}$ model and in Eq. (15) for the N2LOL one.

A plot of the radial functions $y(r), t(r)$, and $Z_{0}(r)$ is given in Fig. 1 of Ref. [31].

\section{THE BRUECKNER-HARTREE-FOCK APPROACH}

The basic ingredient of the BHF approach in nuclear matter $[40,41]$ is the Brueckner reaction matrix $G$ describing the effective interaction between two nucleons in the presence of a surrounding medium. In the case of asymmetric nuclear matter ${ }^{2}$ with neutron density $\rho_{n}$, proton density $\rho_{p}$, total nucleon density $\rho=\rho_{n}+\rho_{p}$, and isospin asymmetry $\beta=$ $\left(\rho_{n}-\rho_{p}\right) / \rho$ (asymmetry parameter), one has different $G$ matrices describing the $n n, p p$, and $n p$ in-medium effective interactions. They are obtained by solving the well known Bethe-Goldstone equation, written schematically as

$$
\begin{aligned}
G_{\tau_{1} \tau_{2} ; \tau_{3} \tau_{4}}(\omega)= & V_{\tau_{1} \tau_{2} ; \tau_{3} \tau_{4}}+\sum_{i j} V_{\tau_{1} \tau_{2} ; \tau_{i} \tau_{j}} \\
& \times \frac{Q_{\tau_{i} \tau_{j}}}{\omega-\epsilon_{\tau_{i}}-\epsilon_{\tau_{j}}+i \varepsilon} G_{\tau_{i} \tau_{j} ; \tau_{3} \tau_{4}}(\omega),
\end{aligned}
$$

where $\tau_{q}(q=1,2, i, j, 3,4)$ indicates the isospin projection of the two nucleons in the initial, intermediate, and final states, $V$ denotes the bare NN interaction, $Q_{\tau_{i} \tau_{j}}$ is the Pauli operator that prevents the intermediate state nucleons $(i, j)$ from being scattered to states below their respective Fermi momenta $k_{F_{\tau}}$, and $\omega$, the so-called starting energy, corresponds to the sum of nonrelativistic energies of the interacting nucleons. The single-particle energy $\epsilon_{\tau}$ of a nucleon with momentum $k$ and mass $m_{\tau}$ is given by

$$
\epsilon_{\tau}(k)=\frac{\hbar^{2} k^{2}}{2 m_{\tau}}+\operatorname{Re}\left[U_{\tau}(k)\right],
$$

\footnotetext{
${ }^{2}$ In the present work we consider spin unpolarized nuclear matter. Spin polarized nuclear matter has been considered, for example, in Ref. [42,43].
} 
where the single-particle potential $U_{\tau}(k)$ represents the mean field felt by a nucleon due to its interaction with the other nucleons of the medium. In the BHF approximation, $U_{\tau}(k)$ is calculated through the so-called on-energy-shell $G$ matrix, and is given by

$$
U_{\tau}(k)=\sum_{\tau^{\prime}} \sum_{k^{\prime}<k_{F_{\tau^{\prime}}}}\left\langle k k^{\prime}\left|G_{\tau \tau^{\prime} ; \tau \tau^{\prime}}\left(\omega=\epsilon_{\tau}(k)+\epsilon_{\tau^{\prime}}\left(k^{\prime}\right)\right)\right| k k^{\prime}\right\rangle_{A},
$$

where the sum runs over all neutron and proton occupied states and the matrix elements are properly antisymmetrized. We make use of the so-called continuous choice [44-46] for the single-particle potential $U_{\tau}(k)$ when solving the Bethe-Goldstone equation. As shown in Refs. [47,48], the contribution of the three-hole line diagrams to the energy per particle $E / A$ is minimized in this prescription and a faster convergence of the hole-line expansion for $E / A$ is achieved [47-49] with respect to the so-called gap choice for $U_{\tau}(k)$.

Once a self-consistent solution of Eqs. (17)-(19) is achieved, the energy per particle can be calculated as

$$
\frac{E}{A}(\rho, \beta)=\frac{1}{A} \sum_{\tau} \sum_{k<k_{F_{\tau}}}\left(\frac{\hbar^{2} k^{2}}{2 m_{\tau}}+\frac{1}{2} \operatorname{Re}\left[U_{\tau}(k)\right]\right) .
$$

\section{A. Inclusion of three-nucleon forces in the BHF approach}

In the microscopic BHF approach the TNFs discussed in the previous section cannot be used directly in their original form. This is because it would require the solution of a three-body Bethe-Faddeev equation in the nuclear medium and currently this is a task still far to be achieved. To avoid this problem an effective density dependent two-body force is built starting from the original three-body one by averaging over the coordinates (spatial, spin and isospin) of one of the three nucleons. The effective NN force due to the NNN one is thus [50,51]:

$$
W(1,2)=\frac{1}{4} \operatorname{Tr}_{\left(\boldsymbol{\tau}_{3}, \boldsymbol{\sigma}_{3}\right)} \int d \mathbf{r}_{3} \sum_{\text {cyc }} W(1,2,3) n(1,2,3) .
$$

In the previous expression $n(1,2,3)$ is the density distribution of the nucleon 3 in relation to the nucleon 1 at $\mathbf{r}_{1}$ and nucleon 2 at $\mathbf{r}_{2}$. The function $n(1,2,3)$ represents the effect of the NN correlations and will suppress the contributions from the short-range part of $W(1,2,3)$. In the following we adopt an usual choice used in literature [50,51]:

$$
n(1,2,3)=\rho g^{2}(1,3) g^{2}(2,3),
$$

where $g(1,3)$ and $g(2,3)$ are the correlation functions between the nucleons $(1,3)$ and $(2,3)$ respectively. The latter quantities can be written as $g(1,3)=1-\eta(1,3)$, where $\eta(1,3)$ is the so-called defect function [and similarly for $g(2,3)]$. Within the BHF approach the defect function should be calculated selfconsistently with the $G$ matrices (17) and the single-particle potentials (19). Thus the average effective two-body force (21) should be calculated self-consistently and added to the bare NN force at each iterative step of the calculations.

To simplify the numerical calculations and following [50,51], in the present work we use central correlation functions $g(i, j)$ independent on spin and isospin. Moreover, it

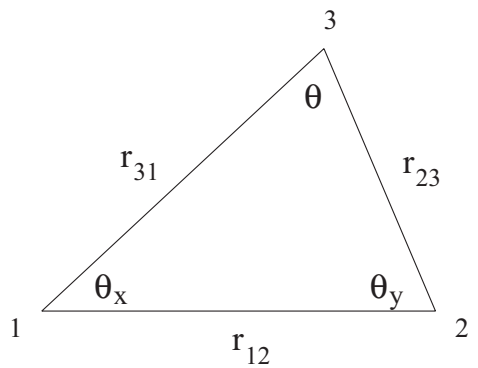

FIG. 2. Coordinates of a three-particle system.

has been shown $[52,53]$ that this central correlation functions, in which are included the main contributions of the ${ }^{1} S_{0}$ and ${ }^{3} S_{1}$ channels, are weakly dependent on the density, and can be approximated $[50,52,53]$ by a Heaviside step function $\theta\left(r_{i j}-r_{c}\right)$, with $r_{c}=0.6 \mathrm{fm}$ in all the considered density range. Note that the average procedure has to be performed for each term involved in the cyclic permutation in Eq. (1).

In the following we report the expressions we used to perform the reduction of the original TNF to the effective density dependent two-body one ([50,51]).

For a generic function $F\left(r_{31}, r_{23}, r_{12}\right)$ where $r_{31}, r_{23}, r_{12}$ are the lengths of the three sides of the triangle as shown in Fig. 2 we have

$$
\begin{aligned}
\int & d \mathbf{r}_{3} \boldsymbol{\sigma}_{1} \cdot \hat{\boldsymbol{r}}_{31} \boldsymbol{\sigma}_{2} \cdot \hat{\boldsymbol{r}}_{31} F\left(r_{31}, r_{23}, r_{12}\right) \cos \theta \\
= & \frac{2 \pi}{3 r_{12}} \int_{0}^{+\infty} d r_{31} \int_{r_{31}+r_{23}}^{\left|r_{31}-r_{23}\right|} d r_{23} r_{31} r_{23} F\left(r_{31}, r_{23}, r_{12}\right) \\
& \times \cos \theta\left[\boldsymbol{\sigma}_{1} \cdot \boldsymbol{\sigma}_{2} \cos \theta+S_{31}\left(\hat{\boldsymbol{r}}_{31}\right) Q\right] \\
\int d & \mathbf{r}_{3} \boldsymbol{\sigma}_{1} \cdot \hat{\boldsymbol{r}}_{31} \boldsymbol{\sigma}_{2} \cdot \hat{\boldsymbol{r}}_{31} F\left(r_{31}, r_{23}, r_{12}\right) \\
= & \frac{2 \pi}{3 r_{12}} \int_{0}^{+\infty} d r_{31} \int_{r_{31}+r_{23}}^{\left|r_{31}-r_{23}\right|} d r_{23} r_{31} r_{23} F\left(r_{31}, r_{23}, r_{12}\right) \\
& \times\left[\boldsymbol{\sigma}_{1} \cdot \boldsymbol{\sigma}_{2} \cos \theta+S_{31}\left(\hat{\boldsymbol{r}}_{31}\right) Q\right], \\
\int & d \mathbf{r}_{3} S_{31}\left(\hat{\boldsymbol{r}}_{31}\right) F\left(r_{31}, r_{23}, r_{12}\right) \\
= & S_{12}\left(\hat{\boldsymbol{r}}_{12}\right) \frac{2 \pi}{3 r_{12}} \int_{0}^{+\infty} d r_{31} \int_{r_{31}+r_{23}}^{\left|r_{31}-r_{23}\right|} d r_{23} r_{31} r_{23} \\
& \times F\left(r_{31}, r_{23}, r_{12}\right) P_{2}\left(\cos \theta_{y}\right),
\end{aligned}
$$

where $\cos \theta=\hat{\boldsymbol{r}}_{31} \cdot \hat{\boldsymbol{r}}_{23}, \quad P_{2}(\cos \theta)=\frac{1}{2}\left(3 \cos ^{2} \theta-1\right), \quad Q=$ $\cos \theta-\frac{3}{2} \sin \theta_{x} \sin \theta_{y}$ (see Fig. 2 for the definition of angles $\theta$, $\theta_{x}$, and $\left.\theta_{y}\right)$, and $S_{31}\left(\hat{\boldsymbol{r}}_{31}\right)=3 \boldsymbol{\sigma}_{3} \cdot \hat{\boldsymbol{r}}_{31} \boldsymbol{\sigma}_{1} \cdot \hat{\boldsymbol{r}}_{31}-\boldsymbol{\sigma}_{3} \cdot \boldsymbol{\sigma}_{1}$.

When we consider the term $W(1,2,3)$, the trace operator acting over $\sigma_{3}$ and $\tau_{3}$ produces a factor 4 on the terms $W_{a}, W_{b}, W_{D}$, while making $W_{d}$ vanish due to the traceless property of the $\sigma$ matrices. On the other hand in the other two cyclic permutation $W(2,3,1)$ and $W(3,1,2)$ all the previous terms make zero because there is always an explicit linear dependence on $\sigma_{3}$ and $\boldsymbol{\tau}_{3}$. For the last term $W_{E}$ we have to discuss separately the $\mathrm{TM}^{\prime}$ and the N2LOL models. The N2LOL model has a dependence on $\boldsymbol{\tau}_{i} \cdot \boldsymbol{\tau}_{j}$ in the term $W_{E}$ so 
TABLE III. Four different parametrizations of the N2LOL threebody force. See text and Ref. [31] for details. The value $c_{1}=$ $-0.00081 \mathrm{MeV}^{-1}$ has been kept fix in all the four cases.

\begin{tabular}{lcccr}
\hline \hline & $c_{3}\left(\mathrm{MeV}^{-1}\right)$ & $c_{4}\left(\mathrm{MeV}^{-1}\right)$ & $c_{D}$ & \multicolumn{1}{c}{$c_{E}$} \\
\hline N2LOL1 & -0.00448 & -0.001963 & -0.5 & 0.100 \\
N2LOL2 & -0.00448 & -0.002044 & -1.0 & 0.000 \\
N2LOL3 & -0.00480 & -0.002017 & -1.0 & -0.030 \\
N2LOL4 & -0.00544 & -0.004860 & -2.0 & -0.500 \\
\hline \hline
\end{tabular}

also in this case only the permutation $W(1,2,3)$ survives. For the $\mathrm{TM}^{\prime}$ model we have instead no isospin dependence in $W_{E}$ so all the three permutations of $(1,2,3)$ give contribution to the effective two-body force.

Using the above formulas we can perform the two-body reduction of the original three-body force $W$ to the effective two-body one. The corresponding expressions for the $\mathrm{TM}^{\prime}$ and the N2LOL models can be found in the AppendixA. The final effective two-body force is finally added to the bare NN interaction and the energy per particle is obtained in the BHF approximation as discussed before.

We want to stress that our average does not take into account some exchange contributions coming from closing a nucleonic fermion line over two different nucleons. These contributions are better evaluated starting from the momentum space form of the three-body potential [4]. In addition, another possible improvement to the average is to close the fermionic line considering an interacting propagator [5]. These tasks are beyond the scope of this work and will be considered in the future.

\section{RESULTS AND DISCUSSIONS}

We now present the results of our calculations of the energy per particle of symmetric nuclear matter (SNM) and pure neutron matter (PNM) using the AV18 NN potential supplemented with the $\mathrm{TM}^{\prime}$ or N2LOL three-nucleon force. Making the usual angular average of the Pauli operator and of the energy denominator [46,54], the Bethe-Goldstone equation (17) can be expanded in partial waves. In all the calculations performed in this work, we have considered partial wave contributions up to a total two-body angular momentum $J_{\max }=9$.

Following Ref. [31], we consider the three parametrizations for the $\mathrm{TM}^{\prime}$ model reported in Table II (hereafter called $\mathrm{TM}^{\prime}$, $\mathrm{TM}^{\prime}$, and $\mathrm{TM}^{\prime}$ ), and the four parametrizations of the N2LOL model reported in Table III (hereafter called N2LOL1, N2LOL2, N2LOL3, and N2LOL4).

In Fig. 3 we show the energy per particle $E / A$ of symmetric nuclear matter. The green double-dash-dotted line, in both panels, represents the energy per particle with no threebody force contribution and using the AV18 NN potential. The resulting saturation point is $\rho_{0}=0.23 \mathrm{fm}^{-3}, E /\left.A\right|_{0}=$ $-16.43 \mathrm{MeV}$, to be compared with the empirical saturation point of nuclear matter, $\rho_{0}=0.16 \pm 0.01 \mathrm{fm}^{-3}, E /\left.A\right|_{\rho_{0}}=$ $-16.0 \pm 1.0 \mathrm{MeV}$ (green box in both panels of Fig. 3). Nuclear matter calculations based on variational chain summation methods [25] give $\rho_{0}=0.30 \mathrm{fm}^{-3}, E /\left.A\right|_{0}=-18.22 \mathrm{MeV}$ in the case of the AV18 potential with no TBF. The differences
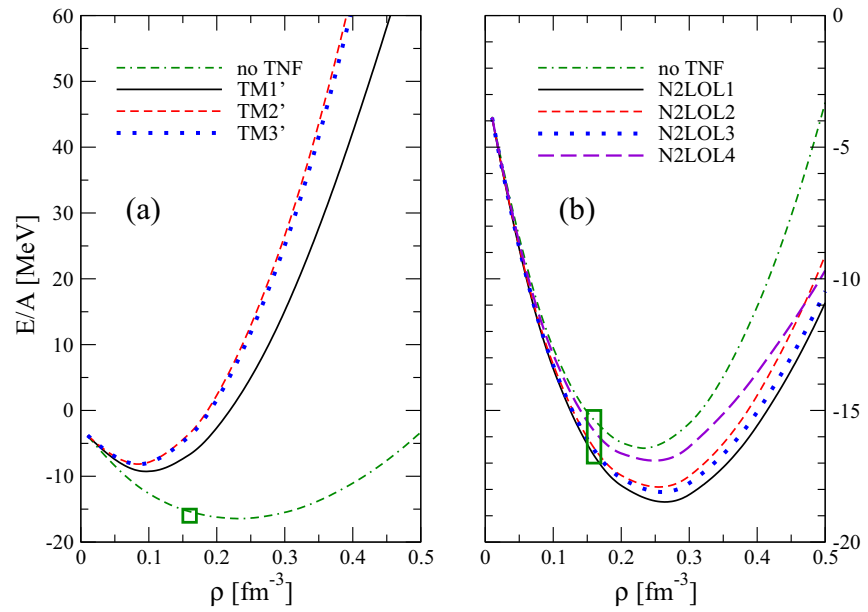

FIG. 3. (Color online) Energy per particle $E / A$ of symmetric nuclear matter as a function of the nucleonic number density $\rho$ for the three parametrizations of the $\mathrm{TM}^{\prime}$ model [panel (a)] and the four parametrizations of the N2LOL one [panel (b)] considered in the present work. The green double-dash-dotted line, in both panels, represents the energy per particle with no three-body force contribution and using the AV18 NN potential. The empirical saturation point of nuclear matter $\rho_{0}=0.16 \pm 0.01 \mathrm{fm}^{-3}, E /\left.A\right|_{\rho_{0}}=-16.0 \pm 1.0 \mathrm{MeV}$ is denoted by the green box in both panels.

between the results of [25] and our BHF results reflect the sensitivity of nuclear matter calculations to the particular quantum many-body technique (see, e.g., $[55,56])$.

We next introduce the three-body forces of Ref. [31] using the average procedure described in the previous section. In the case of the TM' model [Fig. 3(a)] the three-body force produces a strong repulsive effect (i.e., $E / A$ increases with respect to the case with no TNF) in all the considered density range, and shifts the calculated saturation point (see Table IV) to a density lower than the empirical one. At the empirical saturation density $E / A$ increases by $\Delta E=8.7 \mathrm{MeV}(11.5 \mathrm{MeV})$ in the case of the $\mathrm{TM}^{\prime}$ (TM3 ${ }^{\prime}$ ) interaction. At twice the empirical saturation density, i.e., $\rho=0.32 \mathrm{fm}^{-3}, \Delta E=34.8 \mathrm{MeV}$ $(46.2 \mathrm{MeV})$ in the case of the TM1' (TM3') interaction.

The outcome is notably different in the case of the N2LOL [Fig. 3(b)] three-body forces. In this case the TNF produces a decrease of $E / A$ in all the considered density range. At the

TABLE IV. Saturation properties of symmetric nuclear matter for three different parametrizations (first column) of the $\mathrm{TM}^{\prime}$ three-body force. The entry "no TNF" refers to a calculation without three-body force and using the AV18 NN potential. The other entries in the table are the saturation density (second column), the value of energy per particle at saturation (third column), and the value of the symmetry energy at saturation (forth column).

\begin{tabular}{lccc}
\hline \hline & $\rho_{0}\left(\mathrm{fm}^{-3}\right)$ & $E / A(\mathrm{MeV})$ & $E_{\text {sym }}(\mathrm{MeV})$ \\
\hline no TNF & 0.23 & -16.43 & 35.25 \\
TM1 $^{\prime}$ & 0.10 & -9.26 & 22.24 \\
TM2 $^{\prime}$ & 0.09 & -8.15 & 19.96 \\
TM3 $^{\prime}$ & 0.09 & -8.18 & 19.68 \\
\hline
\end{tabular}


TABLE V. Saturation properties of symmetric nuclear matter for three different parametrizations of the N2LOL three-body force.

\begin{tabular}{lccc}
\hline \hline & $\rho_{0}\left(\mathrm{fm}^{-3}\right)$ & $E / A(\mathrm{MeV})$ & $E_{\text {sym }}(\mathrm{MeV})$ \\
\hline N2LOL1 & 0.26 & -18.47 & 42.30 \\
N2LOL2 & 0.25 & -17.90 & 40.02 \\
N2LOL3 & 0.26 & -18.09 & 41.06 \\
N2LOL4 & 0.25 & -16.90 & 36.25 \\
\hline \hline
\end{tabular}

empirical saturation density (at twice the empirical saturation density) $E / A$ decreases by $\Delta E=-1.4 \mathrm{MeV}(-2.9 \mathrm{MeV})$ in the case of the N2LOL1 interaction. The contrasting effect on the energy per particle of SNM of the two TNF models, illustrated in the two panels of Fig. 3, is mainly due to the different action of the repulsive component on the two-body effective force $W(1,2)$ derived from the genuine TNF. As discussed before, the N2LOL model has a nontrivial isospin dependence in the repulsive term $W_{E}$ [see Eq. (8)]. If the parameter $C_{E}$ is positive (negative) the final contribution is repulsive (attractive) on channels with isospin $T=1$ but is attractive (repulsive) on channels with isospin $T=0$. On the other hand in the $\mathrm{TM}^{\prime}$ model there is no isospin dependence on the repulsive part of the three-body force [see Eq. (10)], so $W_{E}$ gives in all channels a repulsive contribution.

The value of the saturation density, energy per particle and symmetry energy at the saturation density are reported in Tables IV and V for the $\mathrm{TM}^{\prime}$ and the N2LOL models respectively. Both models fail to reproduce the empirical saturation point of SNM. This is not surprising (see, e.g., [57]) since in the present nuclear matter calculations we used TNF models [31] whose parameters have been determined to reproduce the properties of light nuclei $(A=3,4)$ and the neutron-deuteron doublet scattering length.

In Fig. 4 we plot our results for the energy per particle of pure neutron matter. The green double-dash-dotted line, in both panels, represents $E / A$ when the TNF is not included. In the case of the $\mathrm{TM}^{\prime}$ model [Fig. 4(a)] the TNF produces a sizable repulsive effect, in all the considered density range, as compared to the case with no TNF. For example, at the empirical saturation density (at twice the empirical saturation density) $E / A$ increases by $\Delta E=9.6 \mathrm{MeV}(38.8 \mathrm{MeV})$ in the case of the TM1' interaction. The effect of TNF on the energy per particle of PNM is less pronounced in the case of the N2LOL model [Fig. 4(b)]. In the particular case of the N2LOL4 parametrization, TNFs make PNM softer with respect to the case where TNFs are not included. Notice that in the case of pure neutron matter, we have just the contribution of the $T=1$ isospin channel and therefore, also for the N2LOL model one has a pure term that provides repulsion. Nonetheless the strength of term associated to repulsion in the $\mathrm{TM}^{\prime}$ model is stronger than the corresponding one for the N2LOL, and consequently a stiffer neutron matter equation of state is obtained.

The nuclear symmetry energy is defined by

$$
E_{\mathrm{sym}}(\rho)=\left.\frac{1}{2} \frac{\partial^{2} E / A}{\partial \beta^{2}}\right|_{\beta=0},
$$
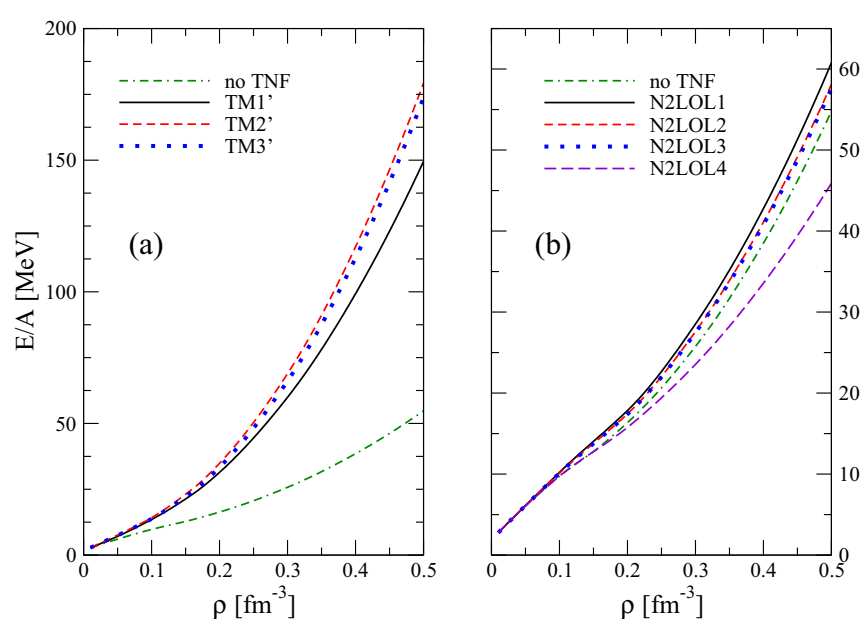

FIG. 4. (Color online) Energy per particle $E / A$ of pure neutron matter as a function of the nucleonic number density $\rho$ for the three parametrizations of the $\mathrm{TM}^{\prime}$ model [panel (a)] and the four parametrizations of the N2LOL one [panel (b)] considered in the present work. The green double-dash-dotted line, in both panels, represents the energy per particle with no three-body force contribution and using the AV18 NN potential.

where $E / A$, the energy per particle of asymmetric nuclear matter, is calculated using Eq. (20) within the BHF approximation.

It has been numerically demonstrated, by various microscopic calculations [58-66] adopting different realistic NN interactions, that the energy per particle of asymmetric nuclear matter can be accurately reproduced by the following relation:

$$
\frac{E}{A}(\rho, \beta)=\frac{E}{A}(\rho, 0)+E_{\mathrm{sym}}(\rho) \beta^{2} .
$$

Thus, in good approximation, the symmetry energy can be expressed in terms of the difference of the energy per particle between symmetric $(\beta=0)$ and pure neutron matter $(\beta=1)$ :

$$
E_{\mathrm{sym}}(\rho)=\frac{E}{A}(\rho, 1)-\frac{E}{A}(\rho, 0) .
$$

The symmetry energy for the $\mathrm{TM}^{\prime}$ and the N2LOL models is shown as function of the density in Figs. 5(a) and 5(b) respectively. The green double-dash-dotted line, in both panels, represents the symmetry energy with no three-body force contribution and using the AV18 NN potential. In Tables IV and V we have reported the values of the symmetry energy for the two TNF models at their respective calculated saturation points $\rho_{0}$ (second column in Tables IV and V).

To compare our results with the value of the symmetry energy extracted from various nuclear experimental data $[67,68]$, we report in Table VI $E_{\text {sym }}$ calculated at the empirical saturation density $\rho_{0}=0.16 \mathrm{fm}^{-3}$ for the different TNF models considered in this work. In the same table, we also report the so-called slope parameter

$$
L=\left.3 \rho_{0} \frac{\partial E_{\mathrm{sym}}(\rho)}{\partial \rho}\right|_{\rho_{0}} .
$$



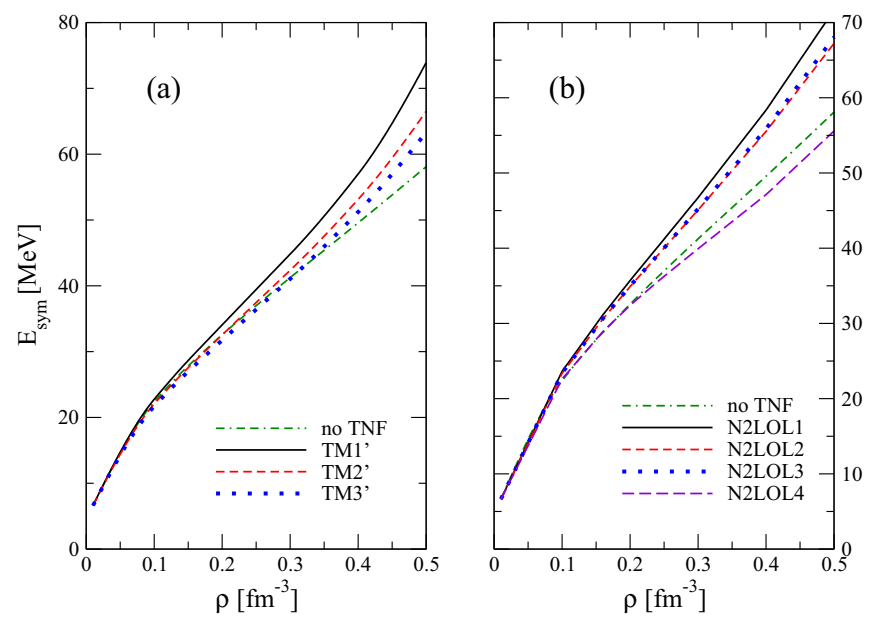

FIG. 5. (Color online) Symmetry energy as a function of the nucleonic number density $\rho$ for the three parametrizations of the $\mathrm{TM}^{\prime}$ model [panel (a)] and the four parametrizations of the N2LOL one [panel (b)] considered in the present work. The green doubledash-dotted line, in both panels, represents the symmetry energy with no three-body force contribution and using the AV18 NN potential.

As we can see (Table VI) our calculated $E_{\text {sym }}$ and $L$ lies within the ranges of values extracted from experimental data [68]: $E_{\text {sym }}\left(\rho_{0}\right)=29.0-32.7 \mathrm{MeV}$, and $L=40.5-61.9 \mathrm{MeV}$.

The TNFs of Ref. [31] have been recently employed for SNM and PNM calculations in [69] using both the variational and the auxiliary field diffusion Monte Carlo approaches. The authors of Ref. [69] used the Argonne V8' [70] NN potential which is a simplified version of the AV18 potential [37], truncated after the linear spin-orbit components and refitted to have the same isoscalar part of the AV18 in all the $S$ and $P$ waves, as well as in the ${ }^{3} D_{1}$ wave and its coupling to the ${ }^{3} S_{1}$ wave. Notice that also in [69] no one of the TNF models was able to reproduce the correct saturation point of symmetric nuclear matter. A direct comparison of our results with those reported in [69] would be ambiguous and inconclusive for the following reasons. First of all, due to the difference in the two-body potential used in the calculations: the AV18 in the present paper and the AV8' in [69]. Second, as discussed in $[55,56]$, the use of different many-body approaches affects considerably the results particularly in the case of SNM.

TABLE VI. Symmetry energy and slope parameter $L$ at the empirical saturation density $\rho_{n m}=0.16 \mathrm{fm}^{-3}$ for different TNF models.

\begin{tabular}{lcc}
\hline \hline & $E_{\text {sym }}(\mathrm{MeV})$ & $L(\mathrm{MeV})$ \\
\hline no TNF & 28.79 & 51.3 \\
TM1' $^{\prime}$ & 29.76 & 52.5 \\
TM2 $^{\prime}$ & 28.58 & 48.7 \\
TM3' & 27.96 & 46.5 \\
N2LOL1 & 31.14 & 56.6 \\
N2LOL2 & 30.59 & 53.7 \\
N2LOL3 & 30.65 & 54.1 \\
N2LOL4 & 28.92 & 44.8 \\
\hline \hline
\end{tabular}

\section{CONCLUSIONS}

A new generation of TNF models has been recently proposed in Ref. [31]. These new TNFs have been used, in conjunction with the Argonne V18 two-nucleon interaction, and their parameters have been determined to simultaneously reproduce the measured binding energies of ${ }^{3} \mathrm{H},{ }^{3} \mathrm{He}$, and ${ }^{4} \mathrm{He}$ nuclei as well as the measured $n$ - $d$ doublet scattering length. A correct prediction for these physical quantities can be regarded as a severe requisite for a realistic nuclear Hamiltonian containing two- and three-nucleon interactions. As shown in Ref. [31], this requirement was not fulfilled by several of the TNF models available in the literature.

In the present work, we have calculated the energy per nucleon of symmetric nuclear matter and pure neutron matter within the BHF approach and using the same nuclear Hamiltonian as the one used in Ref. [31] (i.e., without changing the original values of the TNF parameters) with the purpose of testing this Hamiltonian in a many-body context. We found that none of the TNF models given in [31] is able to reproduce the empirical saturation point of symmetric nuclear matter. This outcome concords with the results obtained with the Urbana VII [71] TNF when used in few-body nuclei and nuclear matter [57] (see also [24]). In particular, in the case of the AV18+TM' Hamiltonian, both the calculated saturation density and the corresponding binding energy per nucleon $(B / A=-E / A)$ are underestimated. The $\mathrm{TM}^{\prime}$ model for the TNF produces a strong repulsive effect both in SNM and PNM, in all the considered density range. In the case of the AV18+N2LOL Hamiltonian, the TNF produces in SNM a decrease of $E / A$ (an increase of the binding energy per nucleon) in all the considered density range.

There can be various reasons why TNFs fitting few-nucleon systems are not able to reproduce the empirical saturation point of SNM. For example, in the BHF approach we are still not able to use the genuine three-body force but, due to the technical reasons explained above, we are forced to include the TNF contribution by performing the average procedure to generate an effective density dependent two-body force. In this way some terms get lost in the average and therefore, in a way, we are not dealing with exactly the same three-body force employed in finite nuclei calculations. An interesting test could be the use of our effective two-nucleon force in finite nuclei calculations. So doing, we may understand at a deeper level the differences between the exact procedure in which we employ the genuine three-nucleon force and our simplified approach that involve an average force. We plan to perform this interesting comparison in a future work. In addition, the inclusion of the exchange terms in the average or the use of a correlation function explicitly dependent from spin and isospin will be definitely an improvement of our calculations. These aspects will be considered in a future work.

\section{ACKNOWLEDGMENTS}

This work has been partially supported by the project PEstOE/FIS/UI0405/2014 developed under the inititative QREN financed by the UE/FEDER throught the program COMPETE"Programa Operacional Factores de Competitividade", and by "NewCompstar", COST Action MP1304. 


\section{APPENDIX}

The explicit expressions for the $\mathrm{TM}^{\prime}$ and the N2LOL models are reported in the following. For the $\mathrm{TM}^{\prime}$ model we have

$$
\begin{aligned}
V^{\mathrm{TM}^{\prime}}\left(r_{12}\right)= & \frac{\rho}{3}\left(\boldsymbol{\tau}_{1} \cdot \boldsymbol{\tau}_{2}\right)\left[\boldsymbol{\sigma}_{1} \cdot \boldsymbol{\sigma}_{2} v_{\sigma}\left(r_{12}\right)+S_{12}\left(\hat{\boldsymbol{r}}_{12}\right) v_{t}\left(r_{12}\right)+v_{r}\left(r_{12}\right)\right], \\
v_{\sigma}\left(r_{12}\right)= & \frac{2 \pi}{r_{12}} \int_{0}^{+\infty} d r_{31} \int_{r_{31}+r_{23}}^{\left|r_{31}-r_{23}\right|} d r_{23} r_{31} r_{23}\left[-\frac{a W_{0}}{3} r_{31} r_{23} y\left(r_{31}\right) y\left(r_{23}\right) \cos \theta+b W_{0} r_{31} r_{23} y\left(r_{31}\right) y\left(r_{23}\right)\right. \\
& \left.+\frac{b W_{0}}{3} r_{31}^{2} r_{23}^{2} \cos ^{2} \theta+\frac{b W_{0}}{3}\left[r_{31}^{2} t\left(r_{31}\right) y\left(r_{23}\right)+r_{23}^{2} t\left(r_{23}\right) y\left(r_{31}\right)\right]\right] g^{2}\left(r_{31}\right) g^{2}\left(r_{23}\right), \\
v_{t}\left(r_{12}\right)= & \frac{2 \pi}{r_{12}} \int_{0}^{+\infty} d r_{31} \int_{r_{31}+r_{23}}^{\left|r_{31}-r_{23}\right|} d r_{23} r_{31} r_{23}\left[-\frac{a W_{0}}{3} r_{31} r_{23} y\left(r_{31}\right) y\left(r_{23}\right) Q\right. \\
& \left.+\frac{b W_{0}}{3}\left[r_{31}^{2} t\left(r_{31}\right) r_{23}^{2} t\left(r_{23}\right) \cos \theta Q+P_{2}\left(\cos \theta_{y}\right) r_{31}^{2} t\left(r_{31}\right) y\left(r_{23}\right)+P_{2}\left(\cos \theta_{x}\right) r_{23}^{2} t\left(r_{23}\right) y\left(r_{31}\right)\right]\right] g^{2}\left(r_{31}\right) g^{2}\left(r_{23}\right), \\
v_{r}\left(r_{12}\right)= & C_{E} W_{0}^{E} \frac{2 \pi}{r_{12}} \int_{0}^{+\infty} d r_{31} \int_{r_{31}+r_{23}}^{\left|r_{31}-r_{23}\right|} d r_{23} r_{31} r_{23}\left[z_{0}\left(r_{31}\right) z_{0}\left(r_{23}\right)+z_{0}\left(r_{31}\right) z_{0}\left(r_{12}\right)+z_{0}\left(r_{12}\right) z_{0}\left(r_{23}\right)\right] g^{2}\left(r_{31}\right) g^{2}\left(r_{23}\right) .
\end{aligned}
$$

For the N2LOL model we have

$$
\begin{aligned}
V^{\mathrm{N} 2 \mathrm{LOL}}\left(r_{12}\right)= & \frac{\rho}{3}\left(\boldsymbol{\tau}_{1} \cdot \boldsymbol{\tau}_{2}\right)\left[\boldsymbol{\sigma}_{1} \cdot \boldsymbol{\sigma}_{2} \tilde{v}_{\sigma}\left(r_{12}\right)+S_{12}\left(\hat{\boldsymbol{r}}_{12}\right) \tilde{v}_{t}\left(r_{12}\right)+\tilde{v}_{r}\left(r_{12}\right)\right] . \\
\tilde{v}_{\sigma}\left(r_{12}\right)= & \frac{2 \pi}{r_{12}} \int_{0}^{+\infty} d r_{31} \int_{r_{31}+r_{23}}^{\left|r_{31}-r_{23}\right|} d r_{23} r_{31} r_{23}\left[-\frac{a W_{0}}{3} r_{31} r_{23} y\left(r_{31}\right) y\left(r_{23}\right) \cos \theta\right. \\
& +b W_{0} r_{31} r_{23} y\left(r_{31}\right) y\left(r_{23}\right)+\frac{b W_{0}}{3} r_{31}^{2} r_{23}^{2} \cos ^{2} \theta+\frac{b W_{0}}{3}\left[r_{31}^{2} t\left(r_{31}\right) y\left(r_{23}\right)+r_{23}^{2} t\left(r_{23}\right) y\left(r_{31}\right)\right] \\
& \left.\left.+\frac{C_{D} W_{0}^{D}}{3}\left[r_{31}^{2} t\left(r_{31}\right) z\left(r_{23}\right)+r_{23}^{2} t\left(r_{23}\right) z\left(r_{31}\right)\right]\right] g^{(} r_{31}\right) g^{2}\left(r_{23}\right), \\
\tilde{v}_{t}\left(r_{12}\right)= & \frac{2 \pi}{r_{12}} \int_{0}^{+\infty} d r_{31} \int_{r_{31}+r_{23}}^{\left|r_{31}-r_{23}\right|} d r_{23} r_{31} r_{23}\left[-\frac{a W_{0}}{3} r_{31} r_{23} y\left(r_{31}\right) y\left(r_{23}\right) Q\right. \\
& +\frac{b W_{0}}{3}\left[r_{31}^{2} t\left(r_{31}\right) r_{23}^{2} t\left(r_{23}\right) \cos \theta Q+P_{2}\left(\cos \theta_{y}\right) r_{31}^{2} t\left(r_{31}\right) y\left(r_{23}\right)+P_{2}\left(\cos \theta_{x}\right) r_{23}^{2} t\left(r_{23}\right) y\left(r_{31}\right)\right] \\
& \left.+\frac{C_{D} W_{0}^{D}}{3}\left[r_{31}^{2} t\left(r_{31}\right) z\left(r_{23}\right) P_{2}\left(\cos \theta_{y}\right)+r_{23}^{2} t\left(r_{23}\right) z\left(r_{31}\right) P_{2}\left(\cos \theta_{x}\right)\right]\right] g^{2}\left(r_{31}\right) g^{2}\left(r_{23}\right), \\
\tilde{v}_{r}\left(r_{12}\right)= & C_{E} W_{0}^{E} \frac{2 \pi}{r_{12}} \int_{0}^{+\infty} d r_{31} \int_{r_{31}+r_{23}}^{\left|r_{31}-r_{23}\right|} d r_{23} r_{31} r_{23}\left[z_{0}\left(r_{31}\right) z_{0}\left(r_{23}\right)\right] g^{2}\left(r_{31}\right) g^{2}\left(r_{23}\right) .
\end{aligned}
$$

[1] N. Kalantar-Nayestanaki, E. Epelbaum, J. S. Messchendorp, and A. Nogga, Rep. Prog. Phys. 75, 016301 (2012).

[2] S. C. Pieper, V. R. Pandharipande, R. B. Wiringa, and J. Carlson, Phys. Rev. C 64, 014001 (2001).

[3] E. Epelbaum, H.-W. Hammer, and U.-G. Meissner, Rev. Mod. Phys. 81, 1773 (2009).

[4] K. Hebeler and A. Schwenk, Phys. Rev. C 82, 014314 (2010); K. Hebeler, S. K. Bogner, R. J. Furnstahl, A. Nogga, and A. Schwenk, ibid. 83, 031301 (2011).

[5] J. W. Holt, N. Kaiser, and W. Weise, Phys. Rev. C 81, 024002 (2010).

[6] R. Machleidt and D. R. Entem, Phys. Rep. 503, 1 (2011).
[7] K. Hebeler, Phys. Rev. C 85, 021002(R) (2012).

[8] H.-W. Hammer, A. Nogga, and A. Schwenk, Rev. Mod. Phys. 85, 197 (2013).

[9] K. Hebeler and R. J. Furnstahl, Phys. Rev. C 87, 031302(R) (2013).

[10] I. Tews, T. Krüger, K. Hebeler, and A. Schwenk, Phys. Rev. Lett. 110, 032504 (2013).

[11] T. Krüger, I. Tews, K. Hebeler, and A. Schwenk, Phys. Rev. C 88, 025802 (2013).

[12] A. Carbone, A. Polls, and A. Rios, Phys. Rev. C 88, 044302 (2013).

[13] A. Carbone, A. Cipollone, C. Barbieri, A. Rios, and A. Polls, Phys. Rev. C 88, 054326 (2013). 
[14] A. Ekström, G. Baardsen, C. Forssén, G. Hagen, M. HjorthJensen, G. R. Jansen, R. Machleidt, W. Nazarewicz, T. Papenbrock, J. Sarich, and S. M. Wild, Phys. Rev. Lett. 110, 192502 (2013).

[15] W. Zuo, I. Bombaci, and U. Lombardo, Eur. Phys. J. A 50, 12 (2014).

[16] J. D. Holt, J. Menéndez, J. Simonis, and A. Schwenk, Phys. Rev. C 90, 024312 (2014).

[17] A. Gezerlis, I. Tews, E. Epelbaum, M. Freunek, S. Gandolfi, K. Hebeler, A. Nogga, and A. Schwenk, Phys. Rev. C 90, 054323 (2014).

[18] L. Coraggio, J. W. Holt, N. Itaco, R. Machleidt, and F. Sammarruca, Phys. Rev. C 87, 014322 (2013); L. Coraggio, J. W. Holt, N. Itaco, R. Machleidt, L. E. Marcucci, and F. Sammarruca, ibid. 89, 044321 (2014).

[19] C. Drischler, V. Soma, and A. Schwenk, Phys. Rev. C 89, 025806 (2014).

[20] A. Roggero, A. Mukherjee, and F. Pederiva, Phys. Rev. Lett. 112, 221103 (2014).

[21] A. Kievsky, S. Rosati, M. Viviani, L. E. Marcucci, and L. Girlanda, J. Phys. G 35, 063101 (2008).

[22] T. Sasakawa and S. Ishikawa, Few-Body Syst. 1, 3 (1986).

[23] J. L. Friar, B. F. Gibson, and G. L. Payne, Phys. Rev. C 37, 2869 (1988).

[24] M. Baldo, I. Bombaci, and G. F. Burgio, Astron. Astrophys. 328, 274 (1997).

[25] A. Akmal, V. R. Pandharipande, and D. G. Ravenhall, Phys. Rev. C 58, 1804 (1998).

[26] Z. H. Li and H.-J. Schulze, Phys. Rev. C 78, 028801 (2008).

[27] N. Chamel, A. F. Fantina, J. M. Pearson, and S. Goriely, Phys. Rev. C 84, 062802(R) (2011).

[28] P. Demorest, T. Pennucci, S. Ransom, M. Roberts, and J. Hessels, Nature (London) 467, 1081 (2010).

[29] J. Antoniadis et al., Science 340, 1233232 (2013).

[30] R. Machleidt, K. Holinde, and Ch. Elster, Phys. Rep. 149, 1 (1987); D. Alonso and F. Sammarruca, Phys. Rev. C 67, 054301 (2003); E. Van Dalen and F. C. Faessler, Nucl. Phys. A 744, 227 (2004).

[31] A. Kievsky, M. Viviani, L. Girlanda, and L. E. Marcucci, Phys. Rev. C 81, 044003 (2010).

[32] S. A. Coon and H. K. Han, Few-Body Syst. 30, 131 (2001).

[33] E. Epelbaum, A. Nogga, W. Glöckle, H. Kamada, Ulf-G. Meißner, and H. Witała, Phys. Rev. C 66, 064001 (2002).

[34] P. Navrátil, Few-Body Syst. 41, 117 (2007).

[35] S. A. Coon and W. Glöckle, Phys. Rev. C 23, 1790 (1981).

[36] H. T. Coelho, T. K. Das, and M. R. Robilotta, Phys. Rev. C 28, 1812 (1983); M. R. Robilotta and H. T. Coelho, Nucl. Phys. A 460, 645 (1986).

[37] R. B. Wiringa, V. G. J. Stoks, and R. Schiavilla, Phys. Rev. C 51, 38 (1995).

[38] D. R. Entem and R. Machleidt, Phys. Rev. C 68, 041001(R) (2003).

[39] A. C. Hayes, P. Navrátil, and J. P. Vary, Phys. Rev. Lett. 91, 012502 (2003); P. Navrátil and W. E. Ormand, Phys. Rev. C 68, 034305 (2003).

[40] B. D. Day, Rev. Mod. Phys. 39, 719 (1967).

[41] M. Baldo, in Nuclear Methods and the Nuclear Equation of State, edited by M. Baldo, International Review of Nuclear Physics Vol. 8 (World Scientific, Singapore, 1999), p. 1.
[42] I. Vidaña and I. Bombaci, Phys. Rev. C 66, 045801 (2002).

[43] I. Bombaci, A. Polls, A. Ramos, A. Rios, and I. Vidaña, Phys. Lett. B 632, 638 (2006).

[44] J. P. Jeukenne, A. Lejeunne, and C. Mahaux, Phys. Rep. 25, 83 (1976).

[45] M. Baldo, I. Bombaci, G. Giansiracusa, U. Lombardo, C. Mahaux, and R. Sartor, Phys. Rev. C 41, 1748 (1990).

[46] M. Baldo, I. Bombaci, L. S. Ferreira, G. Giansiracusa, and U. Lombardo, Phys. Rev. C 43, 2605 (1991).

[47] H. Q. Song, M. Baldo, G. Giansiracusa, and U. Lombardo, Phys. Rev. Lett. 81, 1584 (1998).

[48] M. Baldo, G. Giansiracusa, U. Lombardo, and H. Q. Song, Phys. Lett. B 473, 1 (2000).

[49] M. Baldo, I. Bombaci, G. Giansiracusa, and U. Lombardo, J. Phys. G: Nucl. Part. Phys. 16, L263 (1990).

[50] B. A. Loiseau, Y. Nogami and C. K. Ross, Nucl. Phys. A 165, 601 (1971).

[51] P. Grangé, A. Lejeune, M. Martzolff, and J.-F. Mathiot, Phys. Rev. C 40, 1040 (1989).

[52] M. Baldo and L. S. Ferreira, Phys. Rev. C 59, 682 (1999).

[53] X. R. Zhou, G. F. Burgio, U. Lombardo, H.-J. Schulze, and W. Zuo, Phys. Rev. C 69, 018801 (2004).

[54] P. Grangé, J. Cugnon, and A. Lejeune, Nucl. Phys. A 473, 365 (1987).

[55] I. Bombaci, A. Fabrocini, A. Polls, and I. Vidaña, Phys. Lett. B 609, 232 (2005).

[56] M. Baldo, A. Polls, A. Rios, H.-J. Schulze, and I. Vidaña, Phys. Rev. C 86, 064001 (2012).

[57] R. B. Wiringa, V. Fiks, and A. Fabrocini, Phys. Rev. C 38, 1010 (1988).

[58] I. E. Lagaris and V. R. Pandharipande, Nucl. Phys. A 369, 471 (1981).

[59] I. Bombaci and U. Lombardo, Phys. Rev. C 44, 1892 (1991).

[60] H. Huber, F. Weber, and M. K. Weigel, Phys. Rev. C 51, 1790 (1995).

[61] G. H. Bordbar and M. Modarres, Phys. Rev. C 57, 714 (1998).

[62] C. H. Lee, T. T. S. Kuo, G. Q. Li, and G. E. Brown, Phys. Rev. C 57, 3488 (1998).

[63] W. Zuo, I. Bombaci, and U. Lombardo, Phys. Rev. C 60, 024605 (1999).

[64] W. Zuo, A. Lejeune, U. Lombardo, and J. F. Mathiot, Eur. Phys. J. A 14, 469 (2002).

[65] Kh. Gad and Kh. S. A. Hassaneen, Nucl. Phys. A 793, 67 (2007).

[66] I. Vidaña, C. Providencia, A. Polls, and A. Rios, Phys. Rev. C 80, 045806 (2009).

[67] M. B. Tsang, J. R. Stone, F. Camera, P. Danielewicz, S. Gandolfi, K. Hebeler, C. J. Horowitz, Jenny Lee, W. G. Lynch, Z. Kohley, R. Lemmon, P. Moller, T. Murakami, S. Riordan, X. Roca-Maza, F. Sammarruca, A. W. Steiner, I. Vidaña, and S. J. Yennello, Phys. Rev. C 86, 015803 (2012).

[68] Eur. Phys. J. A 50 (2) (2014), Topical issue on Nuclear Symmetry Energy, edited by B. A. Li, A. Ramos, G. Verde, and I. Vidaña.

[69] A. Lovato, O. Benhar, S. Fantoni, and K. E. Schmidt, Phys. Rev. C 85, 024003 (2012).

[70] B. S. Pudliner, V. R. Pandharipande, J. Carlson, S. C. Pieper, and R. B. Wiringa, Phys. Rev. C 56, 1720 (1997).

[71] R. Schiavilla, V. R. Pandharipande, and R. B. Wiringa, Nucl. Phys. A 449, 219 (1986). 